\title{
Germanium Orthogonal Strip Detectors with Amorphous-Semiconductor Contacts
}

\author{
P. N. Luke ${ }^{1}$, M. Amman ${ }^{1}$, B. F. Phlips ${ }^{2,3}$, W. N. Johnson ${ }^{2}$, and R. A. Kroeger ${ }^{2}$ \\ ${ }^{1}$ Ernest Orlando Lawrence Berkeley National Laboratory, University of California, Berkeley, California 94720 \\ ${ }^{2}$ Naval Research Laboratory, Washington DC 20375 \\ ${ }^{3}$ George Mason University, Fairfax, Virginia 22030
}

\section{Abstract}

Germanium orthogonal strip detectors have been produced using amorphous-semiconductor contacts. The amorphoussemiconductor contact fabrication process is relatively simple, and it is capable of producing fine-pitched electrode structures. The bipolar blocking behavior of the amorphoussemiconductor contact permits its use on both sides of a detector, replacing conventional $\mathrm{B}$ ion implanted and $\mathrm{Li}$ diffused contacts. A $5 \times 5$ orthogonal strip detector has been produced using this technique. Experimental results from this detector are presented.

\section{INTRODUCTION}

Gamma-ray detectors with both high spatial resolution and good energy resolution are desired in a number of applications. One of the applications that we are investigating relates to environmental remediation activities in which a highresolution gamma-ray imager could be used to provide rapid and accurate characterization of radioactivity at contaminated sites. The excellent energy resolution of Ge detectors makes them a good candidate for use in such applications. On the other hand, fabricating position-sensitive Ge detectors with high spatial resolution, especially in two dimensions, has been a major challenge. There has been significant progress over the years on the production of pixel and strip Ge detectors using mostly conventional contact technologies such as B ion implantation and Li diffusion [1-3]. However, the fabrication process using these contact technologies is relatively complex requiring numerous processing steps, and the yield is often low as a consequence. Moreover, the use of Li diffused contacts limits the pitch of contact elements to greater than $1 \mathrm{~mm}$ due to the large initial thickness of such contacts and the risk of electrical shorts from diffusion of $\mathrm{Li}$ when heated or stored at room temperature for an extended period of time. In order to avoid these problems, we have developed a double-sided detector fabrication process based on the use of amorphoussemiconductor contacts.

\section{AMORPHOUS-SEMICONDUCTOR CONTACTS}

The amorphous-semiconductor contact [4] consists of a layer of amorphous $\mathrm{Ge}$ (a-Ge) or amorphous $\mathrm{Si}$ (a-Si) deposited onto the surface of the Ge detector by RF sputtering. A layer of metal is deposited on top of the amorphous layer to form a low-resistance contact area. Multiple contact elements can be realized by forming multiple regions of metallized areas on top of the contiguous amorphous layer. Our earlier measurements indicated that the electrical blocking action of the contact results from a potential barrier formed at the interface between the amorphous-semiconductor layer and the crystalline Ge. The metal layer forms an ohmic contact to the amorphous-semiconductor. Similar contact behavior was observed whether $\mathrm{Al}$ or $\mathrm{Au}$ was used to form the metallized layer.

The use of amorphous-semiconductor contacts for fabricating double-sided, multi-element detectors provides several advantages:

1. Unlike Li-diffused contacts, amorphous-semiconductor contacts are thin $(<1 \mu \mathrm{m})$ and do not suffer from dopant diffusion that can electrically short out closely spaced contacts. This enables small-pitch contacts and thus high spatial resolution to be realized. Also, the significant dead layer associated with the Li contact, which is typically a few hundred microns in thickness, is eliminated.

2. The amorphous-semiconductor contact fabrication process consists of only a few simple steps, and they are all carried out with the device at or near room temperature. In addition, since the amorphous-semiconductor contacts exhibit good blocking behavior under either bias polarity, double-sided electrode structures can be realized using the same process.

3. Besides providing a blocking junction, the amorphoussemiconductor layer also acts as a passivation coating for the Ge surface. This means that the areas between electrodes are automatically passivated as part of the contact fabrication process.

\section{DETECTOR FABRICATION}

A $5 \times 5$ orthogonal strip Ge detector was fabricated using all a-Ge contacts. The detector has an overall size of $25 \mathrm{~mm} \times$ $25 \mathrm{~mm} \times 10 \mathrm{~mm}$ thick. Deep grooves were cut into the Ge crystal to within $\sim 1 \mathrm{~mm}$ from the back surface on four sides (Fig. 1). The grooves define the central active region of the detector, which has an area of $16 \mathrm{~mm} \times 16 \mathrm{~mm}$. The Ge material outside of the grooves is inactive during detector operation and serves as a convenient means for handling the device during processing. The contact fabrication process is described below.

After the detector was cut into the desired geometry, the exposed surfaces of the crystal were lapped with 600 grit followed by 1900 grit lapping compound. The crystal was then polished chemically by etching in a $4: 1 \mathrm{HNO}_{3}: \mathrm{HF}$ mixture until all mechanical damage from cutting and lapping was removed. Immediately prior to loading into the vacuum chamber of the RF sputtering system, the crystal was etched briefly $(\sim 10 \mathrm{~s})$ in the $4: 1$ etchant, quenched in methanol, and 


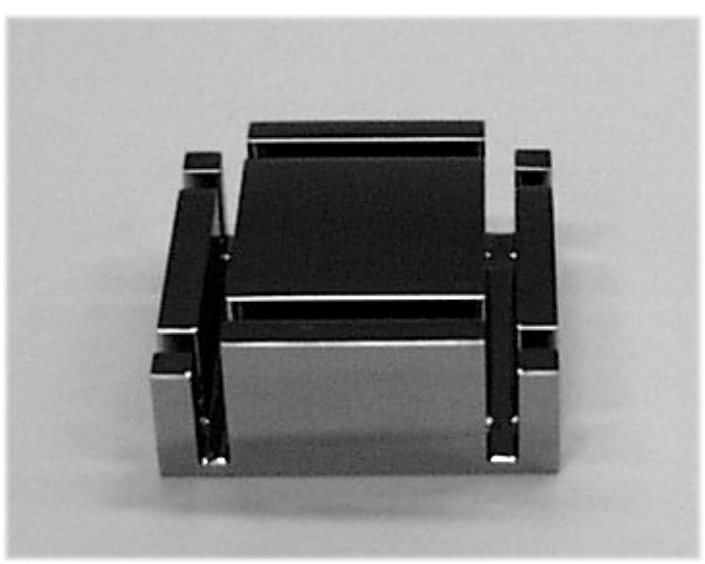

Figure 1. Photograph of the Ge detector crystal prior to contact deposition.

blown dry with nitrogen. Sputter deposition of a-Ge was carried out in pure Ar at a pressure of 7 mtorr. The detector was loaded into the chamber with the bottom of the detector (as viewed in Fig. 1) facing up so that the bottom and side surfaces were coated. The a-Ge deposition rate was $60 \mathrm{~nm} / \mathrm{min}$, and the final a-Ge film thickness used for the present detector was $100 \mathrm{~nm}$. After the a-Ge deposition, the detector was transferred to an evaporation system for $\mathrm{Au}$ deposition. The strip electrodes were defined by evaporating $\mathrm{Au}$ onto the a-Ge layer through a shadow mask. The present design has 5 linear strips at $3 \mathrm{~mm}$ pitch on each side of the detector. The strip width is $2.5 \mathrm{~mm}$, and the gap between strips is $0.5 \mathrm{~mm}$. Two evaporation cycles were used to give a total $\mathrm{Au}$ thickness of about $400 \mathrm{~nm}$. This is the minimum thickness of $\mathrm{Au}$ that was found to be necessary to ensure reliable wire bonding. On this surface (bottom), a guard ring was also formed by masking the central strip region with a square mask and performing another Au evaporation cycle. This guard ring only covers the non-active region of the detector (outside the grooves). After the contacts were deposited, the surface was then protected using picein cement. To form contacts on the other side, the detector was then processed following the same procedure as described above starting with the brief chemical etch prior to loading into the sputtering system. No guard ring was used on this side of the detector. The strip contacts on both sides of the detector extend over the full active area of the detector. After the completion of contact fabrication, the cement layer was removed using TCE. The detector was then rinsed in methanol and blown dry with nitrogen. It should be pointed out that, using appropriate mechanical fixtures, it is possible to sputter coat both sides of the detector with a-Ge first and then perform the metallization on the two sides in tandem. This means that only one final etch prior to sputter deposition is required, and the need for cement masking is eliminated, thereby further simplifying the process.

After completing the contact fabrication, the detector was mounted into an aluminum frame (Fig. 2). Indium foil was used between the detector and the aluminum frame to insure good thermal conduction. The indium contacted the detector on the top surfaces of the part of the crystal outside the grooves. The detector was held against the indium foil using

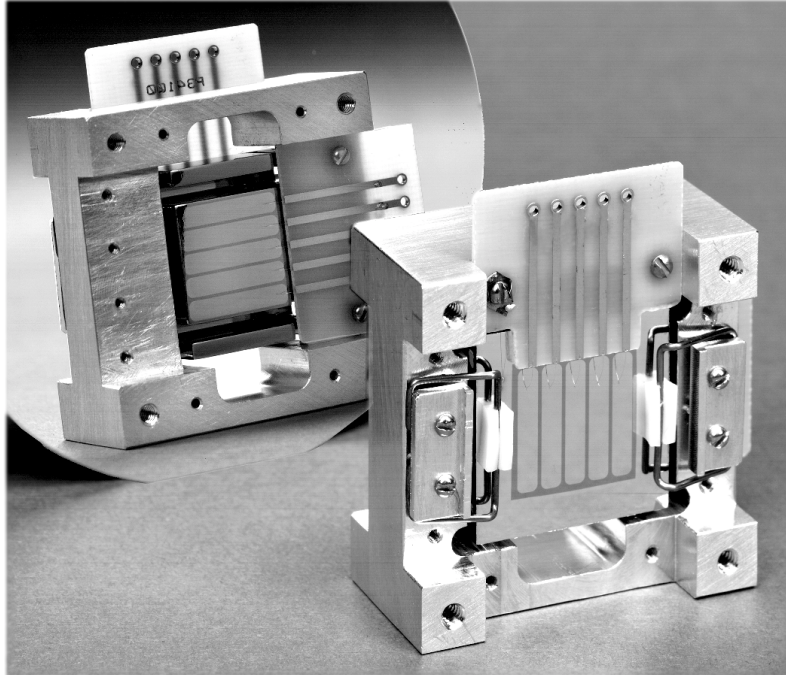

Figure 2. Photograph of a prototype $5 \times 5$ orthogonal strip Ge detector mounted into an aluminum frame.

two spring clips. Two circuit boards, each with 5 circuit traces, were mounted onto the sides of the aluminum frame just above the surfaces of the detector. Electrical connections from the detector electrodes to the circuit traces were made using ultrasonic aluminum wire bonding.

\section{DETECTOR EVAluation}

The detector was cooled and operated in a large volume test cryostat. Each strip was connected through about 12 inches of twisted pair wiring to a hybrid preamplifier $(\mathrm{eV}$ 5093) which operated near room temperature. Each strip on the top side (referenced to Fig. 1) was AC coupled to the preamplifier and biased through a $1 \mathrm{G} \Omega$ resistor. Strips on the opposite face were DC coupled to the preamplifiers and therefore maintained at near ground potential. The guard ring on the bottom side was connected directly to ground. The guard ring serves to define the potential of the inactive region of the detector and isolates the detector mounting points from the active volume. The high-voltage face is isolated from the mounting points by the deep saw cuts. All 10 channels were read out simultaneously into standard NIM shaping amplifiers $(2 \mu$ s shaping time) and CAMAC ADCs. The energy resolution was limited by the preamplifier noise of our system.

The detector was operated at a bias voltage of $+1000 \mathrm{~V}$. Full depletion of the detector occurs at $500 \mathrm{~V}$. Energy spectra were produced for each of the 10 strips. All 5 strips on the high-voltage face (top strips) and the 3 center strips on the ground face (bottom strips) produced good energy resolved spectra, typical of that shown in Fig 3. The two outer bottom strips performed badly due to electric field distortions as discussed below.

A $100 \mu \mathrm{m}$ wide fan-beam of $60 \mathrm{keV}$ gamma rays was scanned across the device. The count rates from the top strips are plotted versus position of the beam in Fig. 4 for irradiation of the top side. The rates were measured in a narrow energy window around $60 \mathrm{keV}$. 


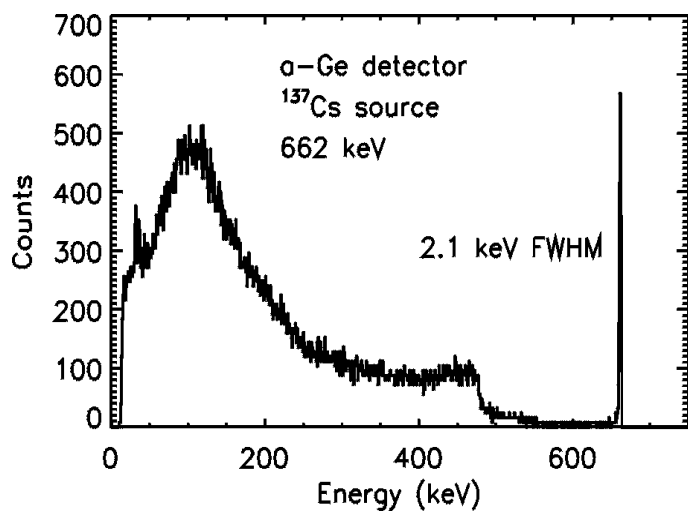

Figure $3 .{ }^{137}$ Cs spectrum typical of each of the strips.

The range of $60 \mathrm{keV}$ gamma rays in Ge is $1 \mathrm{~mm}$, thus the signals in Fig. 4 are for events that occur near the top electrodes. The rate roll off near the outer edges of the outer strips is attributed to distortions in the internal electric field near the edges due to surface channels [5] as discussed below. Introducing a guard ring on this side of the detector should eliminate the edge effect.

Figure 5 shows the response of the bottom strips with the scan beam irradiating again the top side with the scan direction perpendicular to the bottom strips. The strips have the same pitch on both faces. However, the bottom strips appear stretched in the scan plot. This is again attributed to the presence of surface channels, which is depicted schematically in Fig. 6. The holes generated near the top of the detector drift nearly the full distance of the detector thickness, and they are steered toward the inner three strips by the field geometry. For events where holes are collected on the outer two bottom strips, the electrons will be collected at the side surface. As a result, the spectral response on these outer two strips is poor and has a low efficiency. Surface channels are ubiquitously present in virtually all Ge detectors, and they are not necessarily a result of the a-Ge contact technology employed. The deep groove geometry used for this detector may have accentuated the surface channel problem. The a-Ge film along the sides of the grooves has a strong thickness variation because of a shadowing effect during sputter deposition, and this may have contributed to the strong surface channels observed. By reducing or removing the inactive material, the situation may improve substantially. Adding a guard ring of sufficient width would reduce or eliminate the problem. The three central strips (plotted) do have good energy resolution and otherwise behave as expected.

With this detector, signal sharing between strips has been investigated. Events that occur in the gaps between strips split their signals between the two strips. The signal on either strip is roughly proportional to the relative position of the event. Ideally, the sum of the signals on the two strips should equal the total energy. Figure 7 shows events from $122 \mathrm{keV}$ gamma rays that share energy in two bottom strips (designated as strips number 2 and 3 ). The data is taken with uniform illumination of the detector. The events are selected such that two conditions are met: 1) one of the top strips measures the

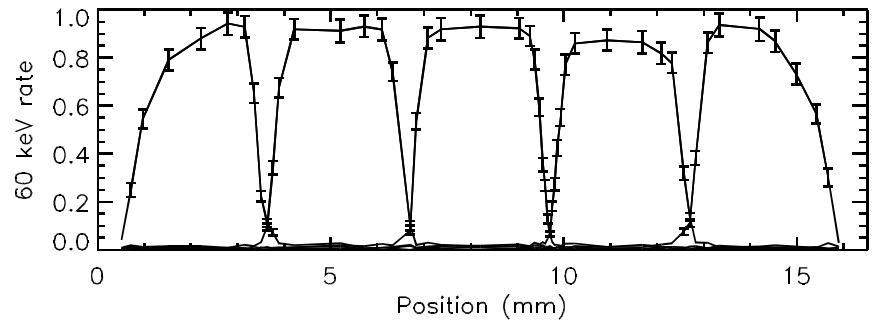

Figure 4. Response of the top strip electrodes as a function of the $60 \mathrm{keV}$ gamma-ray source position.

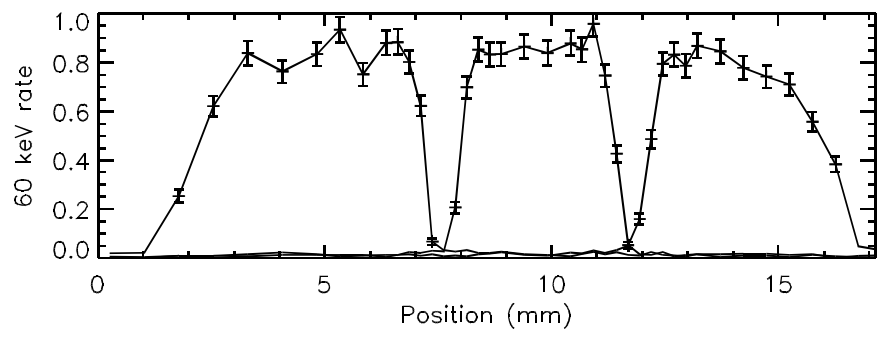

Figure 5. Response of the bottom strip electrodes as a function of the $60 \mathrm{keV}$ gamma-ray source position.

full $122 \mathrm{keV}$ energy deposited, and 2) signals are observed in bottom strips numbers 2 and 3 . The plot indicates that roughly $5 \%$ of the signal is lost in the sum of strips 2 and 3 for events that share roughly equal charge. The magnitude of the lost signal diminishes when the charge is mostly collected on one strip or the other. Careful measurements with the collimated beam (not shown) indicate that the relative signal on the two strips is indeed related to the position of the beam.

The signal loss in Fig. 7 can be understood as the result of field lines in the detector terminating at the surface between the strips. Thus, the charge generated along those field lines is carried directly to the surface of the detector and not to one of the strip electrodes (within the measurement time). For such a situation the induced charge is mostly shared between the two

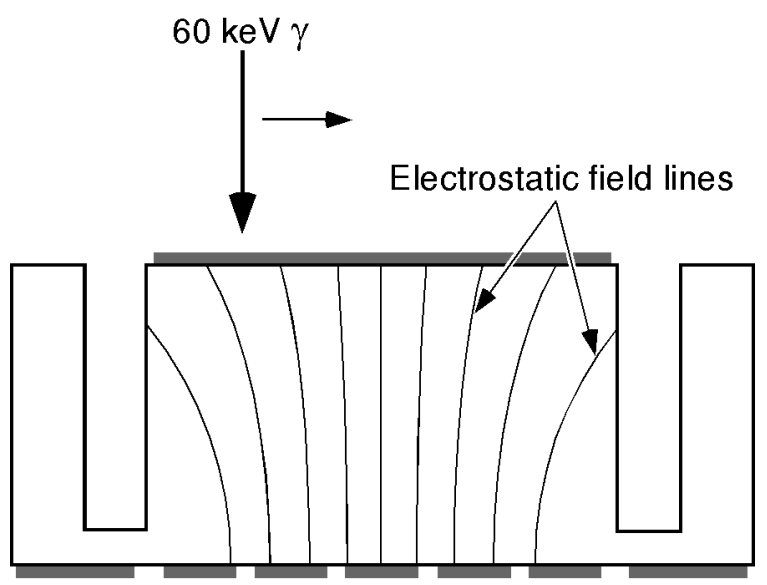

Figure 6. Schematic illustration depicting the electrostatic field lines inside the detector. 


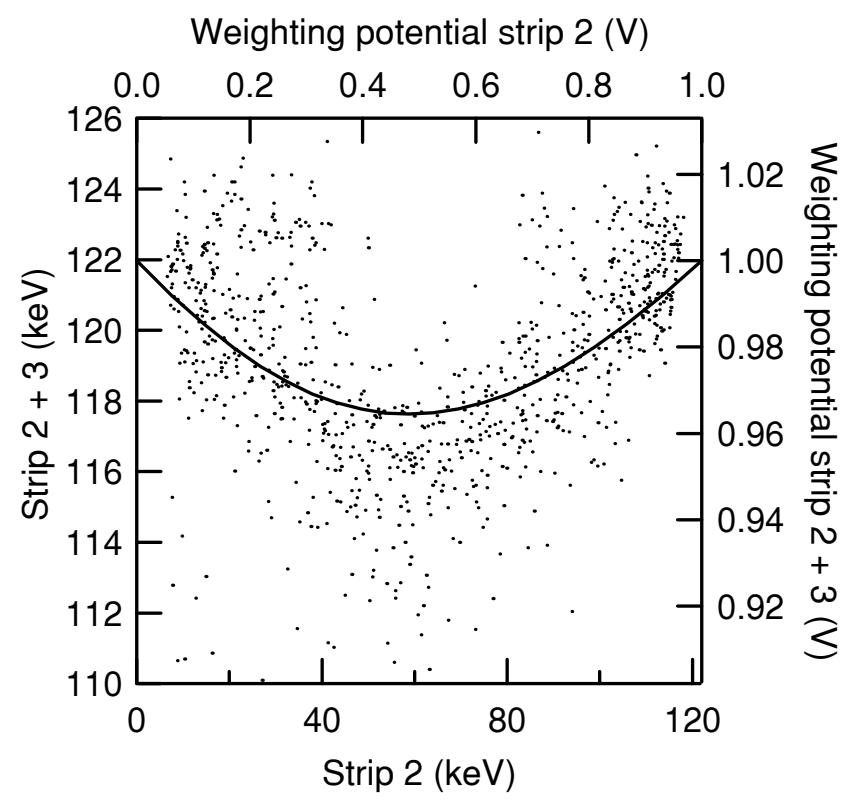

Figure. 7. Scatter plot of $122 \mathrm{keV}$ gamma-ray events that occur in the gap between two strips (dots). For each event the summed energy from the two strips is plotted against the energy from one of the strips. For comparison the summed weighting potential of the two strips is plotted as a function of the weighting potential from one of the strips (solid line). The weighting potentials are those at the detector surface.

strips on each side of the gap, yet the sum of the charges induced on the two strips is slightly less than the full charge since the drifting charge has not been fully collected to either strip. The magnitude of this charge collection deficiency can be calculated using the weighting potential method $[6,7]$. The weighting potential of an electrode is a mathematical construct that simplifies the calculation of the induced charge on that electrode. This potential is a dimensionless quantity numerically equal to the electrostatic potential produced by assuming that the particular electrode of interest is at unit potential, all other electrodes are at zero potential, and no space charge exists. From this potential, the change in the induced charge on the electrode as a result of the movement of a charge $Q$ in the detector is given by $-Q \Delta V_{w}$, where $\Delta V_{w}$ is the difference in the weighting potential between the final and initial locations of the charge $Q$. For the situation of interest here, the electrons generated by a gamma-ray interaction event are fully collected by a top side strip, whereas the holes are collected to the surface between bottom strips 2 and 3 . Consequently, the induced charge on either bottom strip normalized by the full charge will approximately be the weighting potential of that strip evaluated at the location along the detector surface at which the holes are collected. The weighting potential at the surface of the detector between strips 2 and 3 has been determined for both strips 2 and 3 using three-dimensional electrostatic modeling. To compare this model to the measurements, the sum of the two weighting potentials is plotted as a function of one of the weighting potentials and then superimposed on the measured data in Fig. 7. The summed weighting potential plot (solid line) matches reasonably well with the measured data and predicts a maximum charge collection deficiency of about $3.5 \%$ for events that occur midway between the two strips.

\section{CONCLUSIONS}

A prototype $5 \times 5$ orthogonal-strip Ge detector has been fabricated using entirely a-Ge contact technology. The prototype detector operated successfully with no anomalous behavior that could be attributed to the amorphous contacts. Surface channel effects were observed through gamma-ray scanning measurements. Such effects can be minimized or eliminated by adding a guard ring on the active area of the detector. Charge sharing between strips was measured. Loss in the summed signals for events occurring between strips was explained as charge collected at the gap surface. This matches well with weighting potential calculations. The amorphous contact fabrication process is simple and can be easily scaled to larger detectors and much finer strip pitch. We plan to produce a $25 \times 25$ orthogonal strip Ge detector using the same process. The strip pitch will be $2 \mathrm{~mm}$ giving an active area of $5 \mathrm{~cm} \times 5 \mathrm{~cm}$. A guard ring will be employed to minimize surface channel effects.

\section{ACKNOWLEDGMENTS}

This work is supported by the Environmental Management Science Program, Office of Science and Technology, Office of Environmental Management, United States Department of Energy.

\section{REFERENCES}

[1] D. Gutknecht, "Photomask technique for fabricating high purity germanium strip detectors," Nucl. Istrum. Methods Phys. Res., vol. A288, pp. 13-18, 1990.

[2] D. Protic and G. Riepe, "Position sensitive germanium detectors," IEEE Trans. Nucl. Sci., vol. 32, pp. 553-555, 1985.

[3] P. N. Luke, "Gold-mask technique for fabricating segmented-electrode germanium detectors," IEEE Trans. Nucl. Sci., vol. 31, pp. 312-315, 1984.

[4] P. N. Luke, R. H. Pehl, and F. A. Dilmanian, "A 140element Ge detector fabricated with amorphous $\mathrm{Ge}$ blocking contacts," IEEE Trans. Nucl. Sci., vol. 41, pp. 976-978, 1994.

[5] H. L. Malm and R. J. Dinger, "Charge collection in surface channels on high-purity Ge detectors," IEEE Trans. Nucl. Sci., vol. 23, pp. 76-80, 1976.

[6] W. Shockley, "Currents to conductors induced by a moving point charge," J. Appl. Phys., vol. 9, pp. 635-636, 1938.

[7] S. Ramo, "Currents induced by electron motion," Proc. I. R. E., vol. 27, pp. 584-585, 1939. 